\title{
Microarray Analysis reveals that Dietary Retinoic Acid Suppresses Cancer- Related Gene Expression of the Lungs of Cigarette Smoke-Exposed Rats
}

Yuan Xue ${ }^{1}$, Edward P. Meadors ${ }^{2}$, Weiqun Wang ${ }^{1}$ and Richard C. Baybutt ${ }^{1,2 *}$

${ }^{1}$ Department of Human Nutrition, Kansas State University, Manhattan, KS, USA

${ }^{2}$ Applied Health Science Department, Wheaton College, Wheaton, IL, USA

\begin{abstract}
Previously we found that cigarette smoke depleted vitamin A in the lungs and induced tracheal precancerous lesions. To understand the molecular consequences underlying cigarette smoke-induced vitamin A depletion and its associated lung cancer risk, this study investigated the lung cancer-related genes in cigarette smoke-exposed rats with or without dietary retinoic acid, the active metabolite of vitamin A. Twenty-four male weanling rats were fed either a control or a retinoic acid supplemented diet. Half of each group was exposed to 40 commercial cigarettes/ d, $5 \mathrm{~d} /$ wk. After 4 weeks, the rats were sacrificed and their lungs were immediately frozen. Total RNA was extracted and purified, from which cDNA was synthesized and labeled for gene expression analysis. Expressions of 120 genes were measured via a customized microarray. In lungs exposed to cigarette smoke, most of the genes involved in cell division, transcription and cell adhesion were up-regulated. The dietary retinoic acid treatment of the cigarette smoke-exposed lungs was found to down-regulate most of the genes involved in similar functions. In addition, retinoic acid down-regulated four genes, Egr1, Fos, Icam1 and Mmp9, all of which were up-regulated by cigarette smoke. These findings suggest possible molecular mechanisms of cigarette smoke induced-lung cancer and define potential targets of retinoic acid anticarcinogenic actions.
\end{abstract}

Keywords: Cancer; Smoking; Retinoic acid; Cigarette; Genomics

\section{Introduction}

Lung cancer is the leading cause of cancer-related death in both sexes worldwide [1], and in the United States [2]. Most cases of lung cancers are diagnosed after the disease has metastasized. Approaches that target the premalignant stage are needed to reduce the mortality of this disease. Extensive studies have indicated that vitamin A may be a potential agent in lung cancer prevention at an early stage. Vitamin A deficiency has been shown to be associated with bronchial metaplasia and an increased incidence of lung cancer [3]. From our previous study, we have found that exposure to cigarette smoke deceases vitamin A levels in the lungs and results in precancerous lesions in the trachea [4]. The molecular mechanisms by which lung cancer is induced by cigarette smoke and how that is related to vitamin A deficiency is complicated. Numerous components have been suggested to be involved in the process.

Microarray analysis is an effective method of analysis that allows investigators to simultaneously evaluate hundreds to thousands of gene expressions in a single experiment. This method helps identify new or known target genes, or gene function patterns along the lines of the pathways. This technique may prove helpful in determining the molecular mechanisms involved in lung cancer development. Customized microarrays were developed to narrow the focus of a full microarray to specific pathways of interest. In this study, we used customized microarrays to analyze one hundred twenty cancer related genes tailored to our specific research.

The purpose of this study was to identify candidate genes for lung cancer risk and determine whether cigarette smoke changed the expression of these genes. In addition, we determined whether dietary retinoic acid suppressed those changes. Categories of genes related to lung cancer risk were investigated, including: cell division; apoptosis; cell adhesion; tumor suppression. We also determined the gene expression of the retinoic acid receptors as well as growth and transcription factors.

\section{Methods and Materials}

\section{Animals and diets}

Male Sprague-Dawley weanling rats (Charles River Laboratories, Wilmington, MA) weighing about $50 \mathrm{~g}$ was housed individually in stainless steel cages at room temperature under a 12-hrs light: dark cycle (light $600-1800 \mathrm{hrs}$ ) and a relative humidity of 50\%. Animal care and use were approved by the Institutional Animal Care and Use Committee of Kansas State University. Rats were fed a standard AIN$93 \mathrm{G}$ diet [5], with or without additional all-trans retinoic acid $(10 \mathrm{mg} /$ $\mathrm{kg}$ body weight, Sigma, St. Louis, MO). Food intake was recorded daily, and body weight was measured weekly. All rats were pair-fed so that there were no differences in food intake throughout the study.

\section{Cigarette smoke exposure conditions}

Total twenty-four rats were randomly assigned to four groups with six rats per group. Two of those treatment groups were exposed to smoke from two packs of cigarettes per day (nonfiltered commercial cigarettes, 40 cigarettes/ pack). The smoke exposure lasted for 4 weeks, $5 \mathrm{~d} / \mathrm{wk}$. The cigarette smoke treatment has been previously described in detail [4]. Twelve rats were placed together in a plastic chamber measuring $65 \mathrm{~cm}$ long, $50 \mathrm{~cm}$ wide, and $45 \mathrm{~cm}$ high with three holes, two for holding the cigarette at one side of the chamber and another on the opposite

*Corresponding Author: Richard C. Baybutt, Applied Health Science Department, Wheaton College, Wheaton, IL 60187, USA; Tel: +1-630-752-5564; Fax: +1-630752-7277; E-mail: richard.baybutt@my.wheaton.edu

Received May 19, 2012; Accepted June 25, 2012; Published June 30, 2012

Citation: Xue Y, Meadors EP, Wang W, Baybutt RC (2012) Microarray Analysis reveals that Dietary Retinoic Acid Suppresses Cancer-Related Gene Expression of the Lungs of Cigarette Smoke-Exposed Rats. J Nutr Food Sci S2:005. doi:10.4172/2155-9600.S2-005

Copyright: (c) 2012 Xue Y, et al. This is an open-access article distributed unde the terms of the Creative Commons Attribution License, which permits unrestricted use, distribution, and reproduction in any medium, provided the original author and source are credited. 


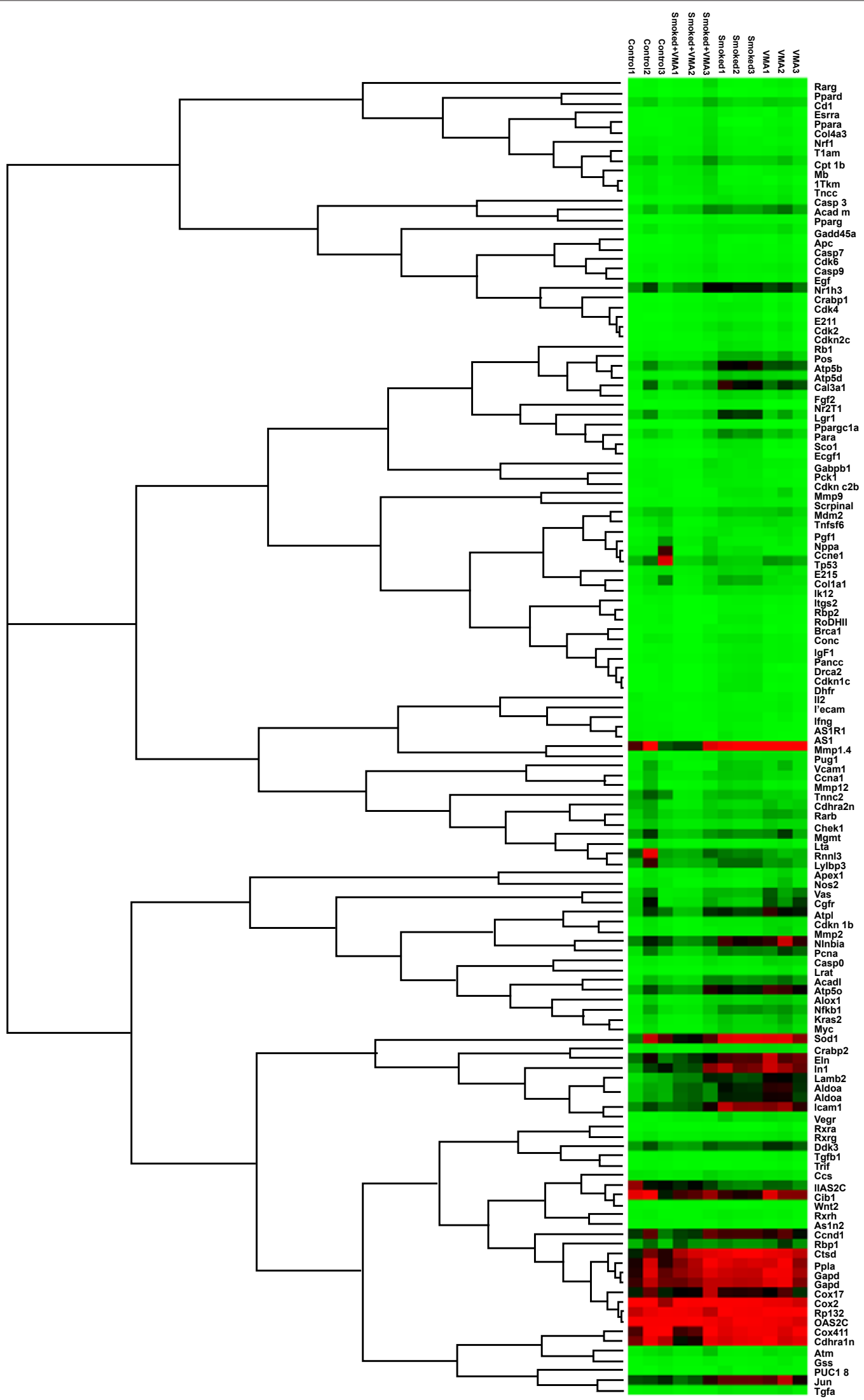

Figure 1: Cluster analyses of the similarities of gene expressions in lungs among different groups. The column represents different treatment groups and the row represents different genes on each array. Red designates that genes were over expressed and green designates that genes were under expressed. 
side of the chamber that was connected to a tube attached to a Leeson vacuum pump (model \# A6C17EB20.1; Labconco, Kansas City, MO) for drawing the smoke. The rats were exposed to approximately 5 min of cigarette smoke (4 cigarettes), followed by 5 min of air, until all the cigarettes assigned to the group were consumed. One pack of cigarettes was considered one session and there was at least two hours of break between sessions. Rats in the control group were placed in another chamber, but were exposed to air only. The extent of exposure of rats to cigarette smoke was ascertained by measuring total particulate matter inhaled, and is reported in our previous study [4].

\begin{tabular}{|c|c|}
\hline Cell cycle genes & $\begin{array}{l}\text { Cell cycle checkpoints: Ccna1, Ccnc, Ccnd1, Ccne1, } \\
\text { Cdk2, Cell cycle arrest: Cdkn1a, Cdkn1b, Cdkn2a, } \\
\text { Ddit3, Gadd45a, Rb1 }\end{array}$ \\
\hline Regulation of cell cycle & $\begin{array}{l}\text { Apc, Atm, Rb1, Brca1, Brca2, Ccnd1, Cdk4, E2f1, } \\
\text { Fgf2, Gadd45b, I1a, I1b, Mdm2 }\end{array}$ \\
\hline Apoptosis genes & $\begin{array}{l}\text { Atm, Bax, Bcl2, Myc, Tnf, Brca1, Casp3, Casp7, } \\
\text { Casp8, Casp9, Ddit3, Mgmt }\end{array}$ \\
\hline p53 Pathway & $\begin{array}{l}\text { Bax, Cdkn1a(p21), E2f1, E2f5, Gadd45a, Igfbp3, } \\
\text { Mdm2, Tnfrsf6, Tp53 }\end{array}$ \\
\hline NFkB Pathway & $\begin{array}{l}\text { Icam1, II2, TNFb, Myc, Nfkb1, Nfkbia, Nos2, Pecam, } \\
\text { Tnf (TNFa), Vcam1 }\end{array}$ \\
\hline Proliferation indicators & Pcna, Fos, Jun \\
\hline Growth factors & Fgf1, Fgf2, Egf, Egfr \\
\hline DNA repair genes & Dhfr, Sod1, Gss \\
\hline Retinoic acid receptors & $\begin{array}{l}\text { Rara, Rarb, Rarg, Rxra, Rxrb, Rxrg, Rbp1, Rbp2, } \\
\text { RoDHII, Crabp1, Crabp2, Lrat }\end{array}$ \\
\hline Other & Kras, Cox2, c-myc, Vegf, EGF, EGFR, Egr1 \\
\hline
\end{tabular}

Table 1: Selected lung cancer-related genes for microarray experiment.

\section{Probe synthesis and microarray hybridization}

The right lobes of the lungs were immediately frozen in liquid nitrogen after harvesting. The day before the RNA extraction, the frozen lung lobe was transferred in solution containing RNAlater (Ambion, Austin, TX) at $4^{\circ} \mathrm{C}$ to prevent RNA degradation. The total RNA was extracted and purified from $150 \mathrm{mg}$ of lung tissue through the use of an RNAeasy Kit (Qiagen, Valencia, CA). The concentration and quality of RNA were checked by Nanodrop spectrophotometer and Agilent 2100 Bioanalyzer (Agilent Technologies, Palo Alto, $\mathrm{CA})$. The probe synthesis was done according to the manufacturer's protocol (SuperArray Bioscience, Frederick, MD). Briefly, $5 \mu \mathrm{g}$ total RNA was used as template for biotinylated cDNA probe synthesis. RNA was reverse-transcribed by gene-specific primers with biotin16-dUTP. Biotinylated cDNA probes were denatured and hybridized to customized gene-specific cDNA fragments spotted on the nylon membranes. The GEArray membranes were then washed and blocked with GEA blocking solution, and incubated with alkaline phosphataseconjugated streptavidin. The hybridized biotinylated probes were detected by chemiluminescent method using the alkaline phosphatase substrate, CDP-Star. The hybridized arrays were visualized using ChemiGlow West on a Fluorochem 8800 (Alpha Innotech Corporation, San Leandro, California) imaging system. The images of arrays are then saved as a grayscale file and ready for the intensity measurement by the software provided by SuperArray.

Each array membrane comprised 120 genes. In addition, five housekeeping genes were included to confirm the integrity of RNA and

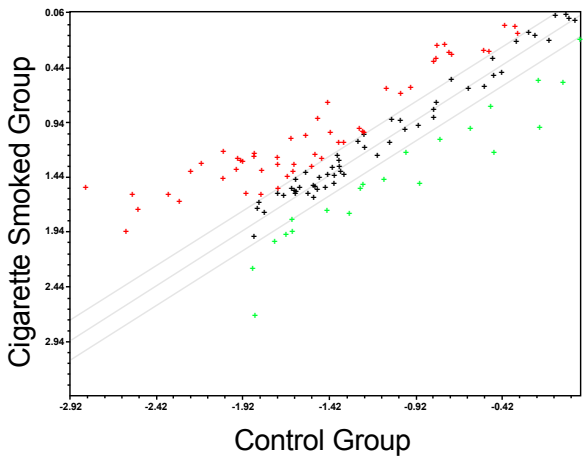

C.

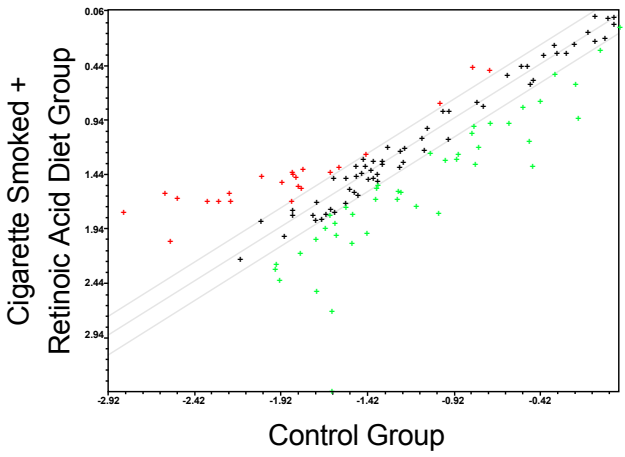

B.

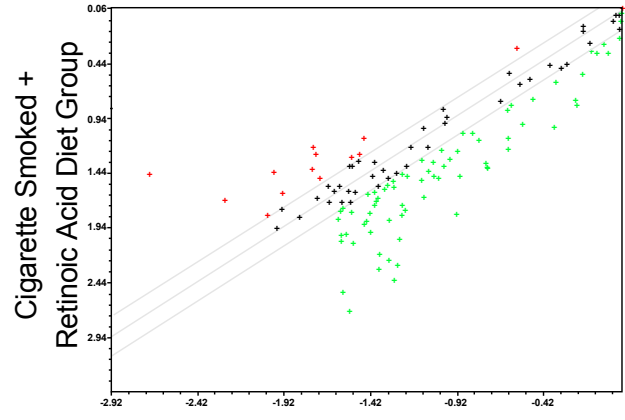

Cigarette Smoke Alone Group

D.

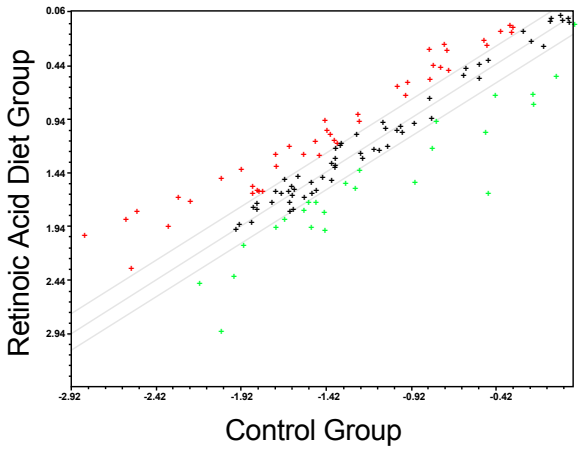

Figure 2: Scatter plot of gene expression of lungs from different groups. Means of triplicate arrays were analyzed for each group. Data are shown as expression levels relative to Gapd, the house keeping gene. Genes above the upper line (red dots) are over expressed, and genes below the lower line (green dots) are under expressed by greater than a 1.5 fold between different groups. A. cigarette smoked group Vs control group; B cigarette smoked+retinoic acid diet group Vs cigarette smoke alone group; C. cigarette smoked+retinoic acid diet group Vs Control; D. retinoic acid diet group Vs control group. 
correct loading of different samples. The selected lung cancer-related genes are listed in Table 1. Twelve customized cDNA GEArrays, three arrays for each group, were purchased from SuperArray.

\section{Microarray data analysis}

Data analysis was performed on all 12 microarrays representing the lungs from different groups. As recommended by Superarray, the image data on each individual microarray was scaled to arbitrary target intensity, using the GEArray Suite software (version 1.1). The intensity of each array was measured by the software. The expression of Gapd, one of the house keeping genes as internal control designed by the company, was consistent for all the arrays showing less than $10 \%$ variation. The Gapd was then chosen for normalization. After all microarrays passing the quality acceptance criteria, normalization was performed using Gapd. The relative expression levels of different genes were estimated by comparing its signal intensity to that of Gapd. Clustering was used to compare the overall gene expression patterns among lungs from different groups.

\section{Statistical analysis}

The normalized data were further transformed to $\log 2$ scale to achieve approximate normality. ANOVA and multiple comparisons were done in SAS PROC GLM to test pre-determined hypotheses. The p-values of the tests were imported into the software Q-value to compute q-values for each test of each gene [6]. The false discovery rate was specified to be 0.05 when calculating the q-values. The tests with q-values smaller than 0.05 were considered significant.

\section{Results}

The similarities of gene expression profiles among different arrays were presented by clustergram (Figure 1). Gene expressions that are more similar are joined at lower heights in the dendrogram and those that are less similar are joined at higher heights. The pairs of genes that showed the most similarities and were joined with lowest height are Cdk2 and cdkn2c, Cdkn1c and Dhfr, Cdkn1b and Ddit2, Fancc and If1, Rbp2 and RoDHII, Tp53 and Ccne1, Fos and Fgf2, Kras2 and Myc.
A 1.5 fold change was chosen to determine the difference of gene expressions among different groups (Figure 2). When the cigarette smoke alone group was compared with control group, most of the genes were overexpressed, with 51 genes overexpressed and 20 under expressed (Figure 2A). When cigarette smoke exposure with retinoic acid diet group was compared with cigarette smoke alone group, majority of the genes were underexpressed. There were 71 underexpressed and 13 genes overexpressed (Figure $2 \mathrm{~B}$ ). When cigarette smoke with retinoic acid diet group compared with the control group, there were 22 genes over-expressed and 44 under-expressed (Figure 2C). The retinoic acid diet treatment alone resulted in 42 genes over-expressed and 24 underexpressed when compared with control group (Figure 2D).

The genes that had greater than 1.5 fold change between two groups were analyzed for statistical significance. All the genes that had significant differences between two groups $(\mathrm{P}<0.05)$ are shown in Table 2. When the cigarette smoke with retinoic acid diet group was compared with the cigarette smoke alone group, 10 genes were significantly down-regulated. For cigarette smoke alone group, 9 genes were significantly changed with 8 up-regulated and 1 down-regulated. Four genes that were up-regulated by cigarette smoke exposure were down-regulated by dietary retinoic acid: Fos, Icam1, Egr1, and Mmp9. When the cigarette smoke plus retinoic acid diet group was compared with control group, 2 genes were up-regulated (cdk4 and Casp7) and 1 was down-regulated (Tp53). The retinoic acid diet alone significantly up-regulated expressions of 3 genes: Casp7, Casp8, and Fos.

The functional classification of the genes that were statistically significant is listed in Table 3 corresponding to Table 2 . The cigarette smoke group vs. the control group resulted in the significant upregulation of 3 cell division genes, 3 transcription factor genes, 1 cell adhesion gene, 1 tumor apoptosis gene, and down-regulated one tumor suppressor gene. When the cigarette smoke with retinoic acid diet group was compared with the cigarette smoke alone group down-regulated genes included 2 cell division genes, 2 growth factor genes, 2 retinoic cell adhesion genes, 1 tumor suppressor inhibitor gene, 1 transcription factor gene, and 2 vitamin A metabolism genes. Cigarette smoke with dietary retinoic acid vs. the control group resulted in the significant

\begin{tabular}{|c|c|c|c|c|c|c|c|}
\hline Symbol & Accession No. & Gene Identity & $\begin{array}{l}\text { Functional } \\
\text { Classification }\end{array}$ & $\begin{array}{l}\text { Smoked vs } \\
\text { Control }\end{array}$ & $\begin{array}{l}\text { Smoked+VitA } \\
\text { vs Smoked }\end{array}$ & $\begin{array}{l}\text { Smoked+VitA } \\
\text { vs Control }\end{array}$ & VitA vs Control \\
\hline Tp53 & NM_030989 & Tumor protein p53 & Tumor suppressor & $\downarrow$ & . & $\downarrow$ & . \\
\hline Mdm2 & XM_235169 & $\begin{array}{l}\text { Transformed mouse } 3 \mathrm{~T} 3 \text { cell double } \\
\text { minute } 2\end{array}$ & $\begin{array}{l}\text { Tumor suppressor } \\
\text { inhibitor }\end{array}$ & . & $\downarrow$ & . & . \\
\hline E2f1 & D63165 & E2F transcription factor 1 & Transcription factor & $\uparrow$ & . & . & . \\
\hline Fos & X06769 & $\begin{array}{l}\text { FBJ murine osteosarcoma viral oncogene } \\
\text { homolog }\end{array}$ & Transcription factor & $\uparrow$ & $\downarrow$ & . & $\uparrow$ \\
\hline Nfkb1 & L26267 & $\begin{array}{l}\text { Nuclear factor of kappa light chain gene } \\
\text { enhancer in B-cells } 1, \text { p105 }\end{array}$ & Transcription factor & $\uparrow$ & & . & . \\
\hline Ecgf1 & NM_001012122 & Endothelial cell growth factor 1 & Growth factor & . & $\downarrow$ & . & . \\
\hline $\operatorname{lgf1}$ & NM_178866 & Insulin-like growth factor 1 & Growth factor & . & $\downarrow$ & . & . \\
\hline Icam1 & NM_012967 & Intercellular adhesion molecule 1 & Cell adhesion & $\uparrow$ & $\downarrow$ & . & \\
\hline Vcam1 & NM_012889 & Vascular cell adhesion molecule 1 & Cell adhesion & . & $\downarrow$ & . & . \\
\hline Egr1 & NM_012551 & Early growth response 1 & Cell division & $\uparrow$ & $\downarrow$ & . & . \\
\hline Cdk4 & NM_031550 & Cyclin-dependent kinase 4 & Cell division & $\uparrow$ & . & $\uparrow$ & . \\
\hline Mmp9 & NM_031055 & Matrix metalloproteinase 9 & Cell division & $\uparrow$ & $\downarrow$ & . & . \\
\hline Rbp2 & NM_012640 & Retinol binding protein 2 , cellular & Vitamin A metabolism & . & $\downarrow$ & . & . \\
\hline RoDHII & BC062000. & Retinol dehydrogenase type II & Vitamin A metabolism & . & $\downarrow$ & . & . \\
\hline Casp7 & NM_022260 & Caspase 7 & Apoptosis & $\uparrow$ &. & $\uparrow$ & $\uparrow$ \\
\hline Casp8 & NM_022277 & Caspase 8 & Apoptosis & . & . & . & $\uparrow$ \\
\hline
\end{tabular}

Table 2: Gene identity and function classification for the statistical significantly expressed genes in the lungs from different groups $(P<0.05)$. Triplicate arrays were analyzed for each group, and data normalized based on Gapd within individual arrays. Dot represents no change between two compared groups. A $\uparrow$ indicates up-regulation and an $\downarrow$ indicates down-regulation. 


\begin{tabular}{|c|c|c|c|c|}
\hline Functional Classification & Smoked vs Control & Smoked+VitA vs Smoked & Smoked+VitA vs Control & VitA vs Control \\
\hline Cell division & $3(+)$ & $2(-)$ & $1(+)$ & . \\
\hline Apoptosis & $1(+)$ & . & $1(+)$ & $2(+)$ \\
\hline Growth factor & . & $2(-)$ & . & . \\
\hline Cell adhesion & $1(+)$ & $2(-)$ & . & . \\
\hline Tumor suppressor inhibitor & . & $1(-)$ & . & . \\
\hline Tumor suppressor & $1(-)$ & . & $1(-)$ & . \\
\hline Transcription factor & $3(+)$ & $1(-)$ & . & $1(+)$ \\
\hline Vitamin A metabolism & . & $2(-)$ & . & . \\
\hline
\end{tabular}

Table 3: Summary of functional classification for the statistical significantly expressed genes in the lungs corresponding to Table 1. Each number indicates the count of statistically significant genes. (+) indicates up-regulation while (-) indicates down-regulation.

up-regulation of 1 cell division gene, 1 apoptosis gene, and the downregulation of 1 tumor suppressor gene. Retinoic acid diet alone vs. the control group found significant up-regulation of 2 apoptosis genes and 1 transcription factor gene.

\section{Discussion}

In this study we investigated the regulation of genes related to cigarette smoke induced-lung cancer risk via customized microarrays and also what role dietary retinoic acid played in such an event in a rodent model. It was observed that cigarette smoke exposure for $4 \mathrm{wk}$ substantially increased the expression of transcription factor genes and genes involved in cell division and apoptosis in the lungs. The dietary retinoic acid decreased the expression of genes involved in the similar functions.

This study provides the first evidence that the gene expression of p53 was down-regulated in cigarette smoke exposed-groups with or without dietary retinoic acid. The p53 gene, a well-known tumor suppressor gene, is especially important to lung carcinogenesis [7]. The loss and inactivation of p53 tumor suppressor functions is an essential contributor to transformation of normal human cells. Mutational alterations of p53 gene occur essentially in 100\% of lung cancer as well [8], indicating that inactivation of p53 pathways occur as early events in the genesis of lung cancer [9]. The decreased gene expression of p53 in response to cigarette smoke suggests that the p53 gene may be a good target marker for early lung cancer detection.

The p53 gene itself is primarily targeted for mutational alterations in lung cancer, and indeed, p53 accumulation is believed to be a dominant step in retinoid-mediated cancer protection effects [10]. Amplification of the Mdm2 gene can accelerate p53 degradation and serve as alternate mechanisms for $\mathrm{p} 53$ functional inactivation [11]. In this experiment the gene expression of $\mathrm{Mdm} 2$ was decreased in the cigarette smoke with retinoic acid diet group when compared with cigarette smoke alone group suggesting that there was less degradation of p53 and therefore a predicted increase in p53. Future experiments measuring protein levels will be critical to ultimately determine how retinoic acid affects p53 in response to smoke exposure.

Another novel finding was that three transcription factors were up-regulated after four weeks of cigarette smoke exposure: E2F transcription factor 1 (E2f1), Fos, and Nuclear factor kappa b (Nfkb1). All three of these transcription factors are shown to be involved in the lung cancer development. E2f1 is a downstream factor of p53 signaling pathway involved in G1 progression of cell cycle. Overexpression of the transcription factor E2f1 correlated with increased tumor growth and aneuploidy via acting as a growth-promoting factor [12]. The Fos is one of the isomers of activator protein-1 (AP-1) that is frequently overexpressed in lung cancer cells [13]. The Nfkbl gene is one of the critical genes that inhibit apoptosis to induce lung cancer [14]. Since the exposure was only four weeks, the up-regulation of these genes may be early events in the development of lung cancer. Among these three transcription factors, only the gene expression of Fos was downregulated in the cigarette smoke with retinoic acid diet group when compared with cigarette smoke alone group. Others have shown that all-trans retinoic acid down-regulates the transcriptional activation by AP-1 through the action of retinoic acid receptor beta [15]. Taken together, these genes may be potential targets for early detection of lung cancer risk and Fos may be a sensitive indicator of the effects of dietary retinoic acid.

Several sets of genes involved in tissue development/growth were also altered by cigarette smoke exposure and dietary retinoic acid. These include a couple of growth factors: Endothelial cell growth factor 1 (Ecgf1), and insulin growth factor 1 (Igf1). The Igf1 is recognized as a potent mitogen for lung cancer cells and there is good evidence that lung cancer cells produce Igf1 [16]. The Ecgf1 gene is a growth factor that promotes angiogenesis and the expression of Ecgf1 is important prognostic factors in lung cancer [17].

Another set of genes is two cell adhesion factors, vascular cell adhesion molecule 1 (Vcam1) and intracellular adhesion molecule 1 (Icam1). The occurrence of aberrations in cell adhesion is a critical phase in the invasion and metastasis of human cancer [18]. The gene expressions of Vcam1 and Icam1 were increased in cigarette smoke alone group but only the level of Icam 1 was decreased when compared with cigarette smoke and dietary retinoic acid group. Therefore dietary retinoic acid may reduce lung cancer risk decreasing to some extent the gene expression of Icam 1 .

The last set of genes involved in tissue growth was cell division molecules, early growth response 1 (Egr1) and cyclin dependent kinase 4 (Cdk4). The increased gene expressions of these two genes in response to cigarette smoke exposure indicate an increase in cell division. Early growth response 1 belongs to a group of proteins that are involved in the progression through G1 phase of the cell cycle following growth factor stimulation. The dietary retinoic acid decreased the gene expression of Egr1 in lungs exposed to cigarette smoke, suggesting that Egr1 may be another target for retinoic acid action on lung cancer prevention. The gene expression of $\mathrm{Cdk} 4$ was increased in cigarette smoked groups and not affected by dietary retinoic acid. Previous research found that cdk4 forms a complex with the D-type cyclins involved in the control of cell proliferation during the G1 phase of cell division, and is believed to play an important role in lung cancer development [19].

It is interesting to note that the gene expression of matrix metalloproteinase 9 (Mmp9) was increased in response to cigarette smoke exposure and dietary retinoic acid decreased the gene expression. The increase of $\mathrm{Mmp}$ is found to be correlated with higher tumor grade and invasiveness [20]. Mmp2 levels are significantly elevated in the serum of patients with metastatic lung cancer, and in those patients 
with high levels, response to chemotherapy is diminished [21]. These findings suggest that certain Mmps might result in an enhanced malignancy. Indeed, Mmp9 which in this study was shown to be upregulated by cigarette smoke and down-regulated by retinoic acid has already been found to be associated with malignant lung cancer tissues [22].

There were decreases of two retinoic acid metabolism genes: retinol binding protein 2 (Rbp2) and retinol dehydrogenase type II (RoDHII) in response to dietary retinoic acid with cigarette smoke exposure compared to smoking. Retinol binding protein 2 binds with retinol and is responsible for the transport of vitamin A within the cell. Retinol dehydrogenase II catalyzes the conversion of retinol to retinal, the first step in retinoic acid synthesis. The decreased expression of both genes suggests that the retinoic acid supplementation decreased the need for transport of retinol to the lung and decreased conversion to retinoic acid.

In summary, the findings from this study suggest several target genes that can be investigated in the future lowering of lung cancer risk induced by cigarette smoke. There was an increase of gene expression of Egr1, Fos, Icam1 and Mmp9 in response to cigarette smoke exposure and decrease with additional dietary retinoic acid, indicating that they may be targets of retinoic acid anticarcinogenic actions. The current study will help define research avenues in the pursuit of the molecular mechanism of lung cancer development.

\section{Acknowledgements}

Supported by Terry Johnson Center for Basic Cancer Research and Kansas Agriculture Experiment Station and College of Human Ecology SRO grant, and by Wheaton College, with its mission to do all things "For Christ and His Kingdom".

\section{References}

1. Parkin DM, Bray FI, Devesa SS (2001) Cancer burden in the year 2000. The global picture. Eur J Cancer 37 Suppl 8: S4-66.

2. Stewart SL, King JB, Thompson TD, Friedman C, Wingo PA (2004) Cancer mortality surveillance--United States, 1990-2000. MMWR Surveill Summ 53: $1-108$

3. Ziegler RG, Mayne ST, Swanson CA (1996) Nutrition and lung cancer. Cancer Causes Control 7: 157-177.

4. Li T, Molteni A, Latkovich P, Castellani W, Baybutt RC (2003) Vitamin A depletion induced by cigarette smoke is associated with the development of emphysema in rats. J Nutr 133: 2629-2634.

5. Reeves PG, Nielsen FH, Fahey GC Jr (1993) AIN-93 purified diets for laboratory rodents: final report of the American Institute of Nutrition ad hoc writing committee on the reformulation of the AIN-76A rodent diet. J Nutr 123 1939-1951.

6. Storey JD, Taylor JE, Siegmund D (2004) Strong control, conservation point estimation, and simultaneous conservative consistency of false discovery rats: A unified approach. J. Royal Statistical Society, Series B 66: 187-205.

7. Cheah PL, Looi LM (2001) p53: an overview of over two decades of study Malays J Pathol 23: 9-16.

8. Sameshima Y, Matsuno Y, Hirohashi S, Shimosato Y, Mizoguchi H, et al. (1992) Alterations of the p53 gene are common and critical events for the maintenance of malignant phenotypes in small-cell lung carcinoma. Oncogene 7: 451-457.

9. Sozzi G, Miozzo M, Donghi R, Pilotti S, Cariani CT, et al. (1992) Deletions of $17 p$ and p53 mutations in preneoplastic lesions of the lung. Cancer Res 52 $6079-6082$.
10. Virmani AK, Gazdar AF (2003) Tumor suppressor genes in lung cancer Methods Mol Biol 222: 97-115.

11. Frederic JK (2001) Molecular biology of lung cancer. Lung cancer 34: S35-S41.

12. Gorgoulis VG, Zacharatos P, Mariatos G, Kotsinas A, Bouda M, et al. (2002) Transcription factor E2F-1 acts as a growth-promoting factor and is associated with adverse prognosis in non-small cell lung carcinomas. J Pathol 198: 142156

13. Maeno K, Masuda A, Yanagisawa K, Konishi H, Osada H, et al. (2006) Altered regulation of $\mathrm{c}$-jun and its involvement in anchorage-independent growth of human lung cancers. Oncogene 25: 271-277.

14. Batra RK, Guttridge DC, Brenner DA, Dubinett SM, Baldwin AS, et al. (1999) IkappaBalpha gene transfer is cytotoxic to squamous-cell lung cancer cells and sensitizes them to tumor necrosis factor-alpha-mediated cell death. Am J Respir Cell Mol Biol 21: 238-245

15. Altucci L, Gronemeyer H (2001) The promise of retinoids to fight against cancer Nat Rev Cancer 1: 181-193.

16. Unsal E, Koksal D, Yurdakul AS, Atikcan S, Cinaz P (2005) Analysis of insulin like growth factor 1 and insulin like growth factor binding protein 3 levels in bronchoalveolar lavage fluid and serum of patients with lung cancer. Respir Med 99: 559-565.

17. Giatromanolaki A (2001) Prognostic role of angiogenesis in non-small cell lung cancer. Anticancer Res 21: 4373-4382.

18. Murakami Y, Sekiya T (1998) Accumulation of genetic alterations and their significance in each primary human cancer and cell line. Mutat Res 400: 421 437.

19. Petty WJ, Dragnev KH, Dmitrovsky E (2003) Cyclin D1 as a target for chemoprevention. Lung Cancer 41 Suppl 1: S155-161.

20. Liotta LA, Kohn EC (2001) The microenvironment of the tumour-host interface. Nature 411: 375-379.

21. Ruddon RW (1995) Cancer biology. (3rdedn), New York: Oxford University Press.

22. Fiorelli A, Rizzo A, Messina G, Izzo A, Vicidomini G, et al. (2012) Correlation between matrix metalloproteinase 9 and 18F-2-fluoro-2-deoxyglucose-positron emission tomography as diagnostic markers of lung cancer. Eur J Cardiothorac Surg 41: 852-860.
Submit your next manuscript and get advantages of OMICS Group submissions

Unique features:

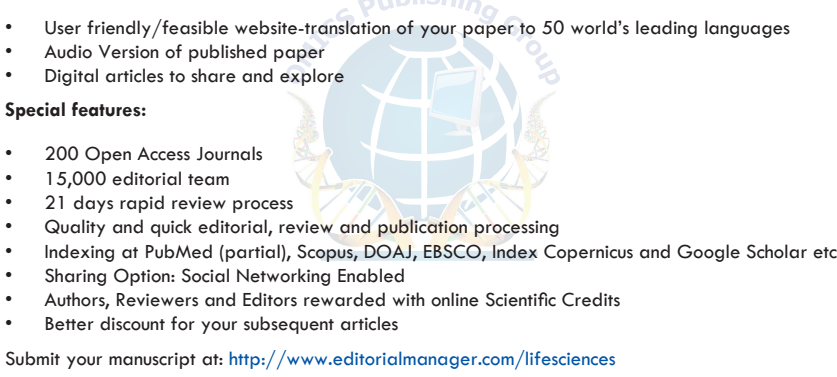

This article was originally published in a special issue, Dietary factors for cancer prevention handled by Editor(s). Dr. Weiqun (George) Wang, Kansas State University, Barton 